\title{
Influencia del agua en el desempeño de los pavimentos: lluvia ácida
}

\author{
Influence of water in the performance \\ of road surface: acid rain
}

\section{Carlos Javier Obando Gamboa}

Universitaria Agraria de Colombia UNIAGRARIA. Bogotá, Colombia

Correo electronico: iccobando@gmail.com 


\section{Resumen}

El agua superficial contribuye en la oxidación del asfalto, más aún cuando ingresa por los poros de la capa de rodadura, sin embargo, el mayor efecto destructivo se manifiesta en forma combinada con las cargas del tráfico ya que el agua alojada en las fisuras, poros e intersticios del pavimento por efecto de la presión de los neumáticos, genera una presión de vacíos que gradualmente destruye el pavimento asfáltico. Es conocido que diferentes agentes químicos pueden modificar las características mecánicas y desempeño de los concretos asfálticos, esta es la base para el uso y desarrollo de diferentes modificadores con el objeto de mejorar el comportamiento del asfalto frente a diferentes solicitudes y/o factores, así mismo las adiciones y aditivos en los pavimentos hidráulicos. Partiendo del hecho que existen agentes químicos y que el agua superficial puede infiltrarse en el concreto asfáltico e hidráulico, y que ésta sometida a fuerzas externas causarán inevitables esfuerzos internos adicionales, podemos inferir que los agentes químicos presentes en el agua de escorrentía o agua lluvia podrían verse potencializados, modificar el comportamiento mecánico de los concretos utilizados en los pavimentos, acelerar los procesos de envejecimiento y causar daños estructurales

\section{Palabras \\ Claves: \\ lluvia ácida, modificadores, presione}




\section{Abstract}

Surface water contributes to oxidize asphalt, even when it comes in through the pores of the granular sub base, however, the major destructive effect comes out in a mixed form with the loads of traffic since the water kept in the fissures, pores and clefts of the road

Keywords

Acid rain, modifiers, pressures. surface due to the pressure of pneumatics, generates a vacuum pressure that gradually destroys the asphalt of the road. It is well known that different agents can modify the mechanical characteristics and the performance of asphalt concrete; this is the base for the usage and development of different modifiers with the aim of improving the behavior of asphalt when facing different applications or factors, just in the same way as additions and additives in hydraulic paving. Starting from the fact that chemical agents do exist and surface water can get through asphaltic and hydraulic concrete, and trhe fact that they are submitted to external forces that will cause additional unavoidable internal efforts, we can infer that the chemical agents present in the water drained from the rain could be enhanced, could modify the mechanical behavior of the concrete used in paving, and could also speed up the aging of the road surface and cause structural damages. 


\section{Introducción}

Cuando se habla de agua y carreteras, aparecen conceptos y problemas complejos en el entorno. Ciertamente, el agua es necesaria en los procesos de construcción, pero durante la vida útil de un pavimento es imperativo tomar las medidas necesarias para mantenerla alejada de la estructura de la carretera o controlar sus niveles para no vulnerar el equilibrio. Para esto se diseñan y construyen obras para la evacuación de aguas como cunetas, drenes y alcantarillas, entre otras, así como mecanismos de bombeo sobre la capa de rodadura. También se utilizan materiales como geotextiles para mejorar la evacuación y control de la humedad. A pesar de estas precauciones, el agua hace contacto con el pavimento y causa daños en los granulares al entrar por la carpeta de rodadura o por succión, cuando se trata de agua freática. Esto afecta estructuralmente a la carpeta de rodadura; y, por otra parte, los daños por oxidación y presiones internas cuando el agua se infiltra a través de los granulares a la rodadura o por la escorrentía superficial, producen daños en el comportamiento mecánico del concreto asfáltico e hidráulico.

En este documento se enfatiza en el efecto del agua sobre la carpeta de rodadura asfáltica, así como de los agentes químicos presentes en esta debido a la polución o lluvia ácida. Esta última ocurre cuando la humedad en el aire se combina con los óxidos de nitrógeno y el dióxido de azufre emitidos por la industria y los vehículos, dando origen al ácido sulfúrico y ácidos nítricos.

Los efectos de la lluvia ácida sobre los concretos asfálticos serán analizados hipotéticamente, tomando como referencia los resultados de pruebas con modificadores a base de azufre, información mundial sobre el efecto de la lluvia ácida en las edificaciones, y reacciones químicas que se producen entre algunos componentes de los agregados, como el carbonato de calcio y la lluvia ácida. 


\section{Lluvia acida}

Como ya se ha dicho, la lluvia ácida es un fenómeno resultante de la combinación de la humedad presente en el aire con los óxidos de nitrógeno y el dióxido de azufre emitidos por fábricas, centrales eléctricas y vehículos que queman carbón o productos derivados del petróleo; estos químicos, en interacción con el vapor de agua, forman ácido sulfúrico y ácidos nítricos, y finalmente estas sustancias químicas caen a la tierra a través de las precipitaciones.

Los contaminantes atmosféricos primarios que dan origen a la lluvia ácida pueden recorrer cientos o miles de kilómetros, en virtud de los vientos, antes de precipitarse en forma de rocío, lluvia, llovizna, granizo, nieve, niebla o neblina. Cuando la precipitación se produce, puede provocar importantes deterioros en el ambiente (Figura 1) [1-3].

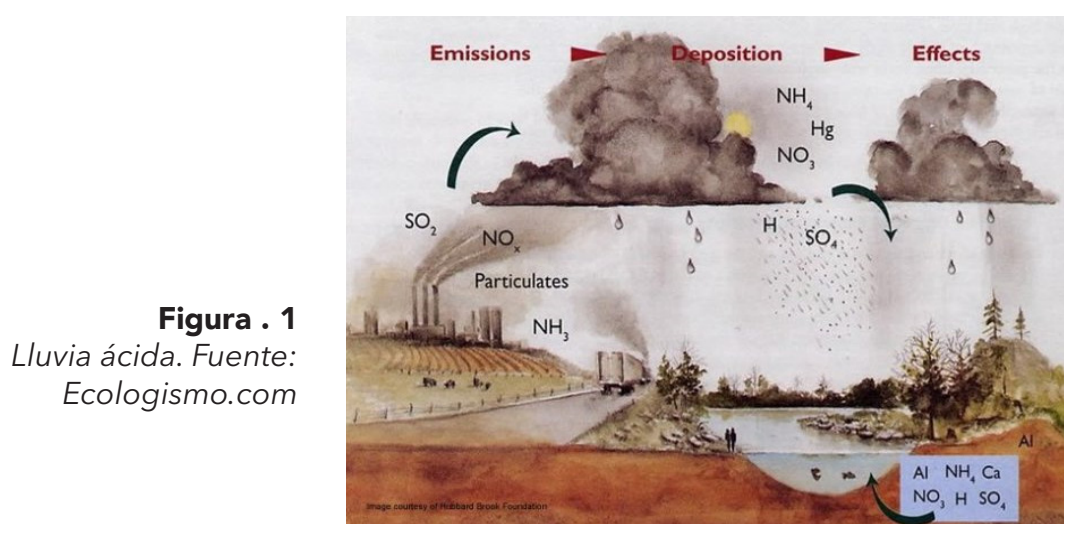

El agua de la lluvia común presenta un $\mathrm{pH}$ ligeramente ácido de aproximadamente 5.65, debido a la presencia del CO2 atmosférico que forma ácido carbónico, $\mathrm{H} 2 \mathrm{CO} 3$. Por tanto, se puede considerar lluvia ácida si se encuentra un $\mathrm{pH}$ de menos de 5.00; sin embargo, la lluvia ácida puede alcanzar un pH de inclusive 3.0, igual que el del vinagre. Estos valores de $\mathrm{pH}$ se alcanzan por la presencia de compuestos como el ácido sulfúrico, $\mathrm{H} 2 \mathrm{SO} 4$, y el ácido nítrico, HNO3. 


\section{Efectos de la lluvia acida}

La acidificación de las aguas de lagos, ríos y mares dificulta el desarrollo de vida acuática en estas aguas, lo que aumenta en gran medida la mortalidad de peces. Igualmente, acaba con los microorganismos fijadores de nitrógeno y afecta directamente a la vegetación, por lo que produce daños importantes en las zonas forestales.

El término "lluvia ácida" abarca la sedimentación tanto húmeda como seca de contaminantes ácidos que pueden producir el deterioro de la superficie de los materiales, más concretamente, por su carácter corrosivo, la lluvia ácida deteriora las construcciones y las infraestructuras. Puede disolver, por ejemplo, el carbonato de calcio, $\mathrm{CaCO} 3$, y afectar de esta forma a los monumentos y edificaciones construidas con mármol o caliza [3].

En Colombia, es común la utilización de piedra caliza y arenisca como agregados para las mezclas asfálticas, debido a su buena adherencia con el asfalto y a la conformación de materiales granulares, pues estos materiales abundan a lo largo y ancho del territorio nacional; sin embargo, la infraestructura construida con caliza y arenisca (carbonática) presenta un deterioro denominado el "mal de piedra", originado por el ácido sulfúrico de la lluvia ácida, que al reaccionar con el carbonato de calcio produce sulfato de calcio, sustancia que el agua de lluvia disuelve y arrastra, así:

$\mathrm{CaCO} 3$ (piedra caliza) + H2SO4 (lluvia ácida) ----------> CaSO4 (yeso) + CO2 $+\mathrm{H} 2 \mathrm{O}$ 
Es decir, la caliza se transforma en yeso, y este es disuelto por el agua con mucha mayor facilidad. Además, al tener un volumen mayor, el yeso actúa como una cuña, provocando el desmoronamiento y disolución de la piedra.

Las precipitaciones de lluvia ácida no se han medido con exactitud, pero por lo general caen al principio de la temporada invernal, con un tiempo de caída variable, que depende de cuánto gas tóxico hay en la atmósfera, pero en sí, la lluvia ácida podría durar sólo unos minutos y limitarse al comienzo de un aguacero 
El impacto adverso de la infiltración de lluvia en la estructura de pavimento asfáltico no debe ser ignorado, y más si se tiene en cuenta que, según la regla de infiltración del agua, en condiciones de lluvia, el agua penetra continuamente en la capa de rodadura, haciendo que el contenido volumétrico de agua en la superficie se incremente gradualmente hasta la saturación, y que, una vez esta deja de caer, la infiltración del agua es constantemente expulsada hacia fuera de la estructura, dado que el contenido volumétrico de agua sigue en aumento por un corto tiempo adicional, para luego disminuir gradualmente. Se puede afirmar, entonces, que un pavimento asfáltico es sometido de manera habitual a saturaciones relativamente prolongadas, es decir, que el tiempo durante el cual el asfalto está en contacto con el agua es alto, lo que podría generar altos índices de oxidación en la mezcla.

Se ha determinado que el agua alojada en las fisuras del pavimento, combinada con el efecto de la presión de los neumáticos, genera una presión de poros al interior del pavimento que lo destruye del interior hacia el exterior.

De otro lado, es normal que en ambientes húmedos o saturados, las mezclas reporten disminución en la cohesión de los asfaltos con el granular, lo cual afecta la estabilidad y rigidez Marshall en las mezclas asfálticas.

Los anteriores fenómenos, sumados al efecto destructivo de los ácidos presentes en el agua de escorrentía (lluvia ácida), pueden disminuir la vida útil de un pavimento asfáltico, por las siguientes razones:

- El concreto asfáltico saturado con agua ácida (lluvia), además de la oxidación, puede sufrir un desgaste externo adicional, de modo que los agregados que contienen carbonato de calcio en su composición pueden deteriorarse y lavarse con el tiempo.

- La disminución en el perímetro de los agregados superficiales como consecuencia de la lluvia ácida puede causar el desprendimiento de los agregados. facilitando la intrusión de agua en la matriz asfáltica [3]. 
- Dado que por efecto de la lluvia ácida, los agregados pueden perder diámetro, el asfalto a su alrededor pierde la capacidad aglutinante y, por consiguiente, pueden presentarse problemas de adherencia, no química (aunque debe estudiarse) sino mecánica, como se indica en la Figura 2.

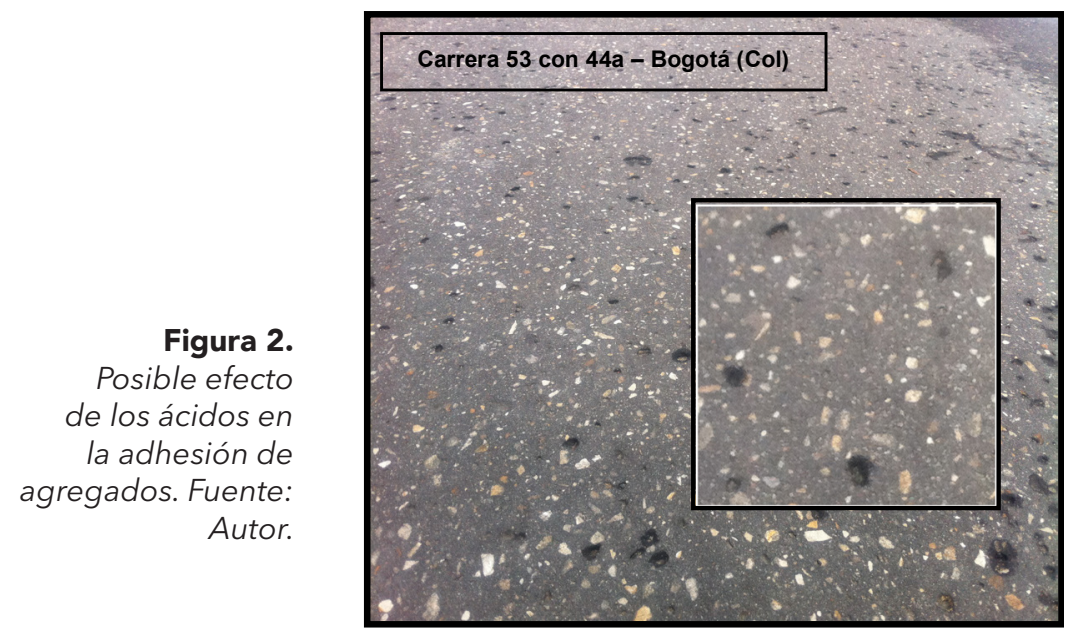

- Cuando el agua ácida penetra el asfalto y se combina con las cargas cíclicas ocasionadas por el paso de los vehículos, la intrusión continua de la misma, se acelera, dado que las presiones internas sobre los poros del asfalto crean un efecto "lavadora", y esto propicia el efecto corrosivo y desgastante interno de la estructura del asfalto, ocasionando poros cada vez mas grandes (huecos) por donde el agua entra más fácilmente.

- El agua bombeada hacia afuera del asfalto arrastra gran cantidad de material de caliza convertido en yeso, lo cual ocasiona huecos cada vez más grandes en la matriz asfáltica, que combinados periódicamente con las cargas del tránsito, produce roturas en el concreto. 
- Como el agua entra cada vez con mayor facilidad en la estructura del pavimento, se acelera el proceso de oxidación, lo cual rigidiza al concreto. En estas circunstancias, los vacios ya creados en el interior del asfalto producto del lavado (desintegración de los agregados, por el "mal de piedra") hacen que el asfalto se agriete con mayor facilidad (velocidad).

- Por último, dado que el agua de escorrentía también se infiltra por el suelo circundante a la estructura de pavimento, es natural que las capas granulares de este se vean afectadas por el efecto de la lluvia ácida. Como se dijo antes, dado lo anterior, los agregados con presencia de carbonato de calcio (caliza y arenisca) se reblandecen poco a poco y se lavan en los flujos de agua freática, lo que con el tiempo generará vacíos bajo la rodadura, ocasionando deformaciones evidentes.

El efecto del azufre presente en la lluvia ácida merece un capítulo aparte, en particular cuando el asfalto no presenta agregados con composición carbónica, es decir, cuando se cuenta con agregados como el granito. En este sentido, existen modificadores a base de azufre que transforman los asfaltos y causa diferentes comportamientos mecánicos.

\section{Modificadoes a base de azufe}

Dependiendo de la constitución química del asfalto, aproximadamente del $15 \%$ al $18 \%$ de azufre puede ser dispersado en él y permanecer estable por un periodo prolongado. En la Figura 3, se ilustra la influencia del contenido de azufre sobre el ensayo Marshall de un concreto asfáltico-arena, usando un asfalto de penetración 200, caso en el que se presenta un incremento sustancial en la estabilidad Marshall. También se ha establecido que el 
azufre mezclado en el asfalto actúa como un diluyente. Hay otro proceso que utiliza una gran cantidad de azufre y que se comporta como un filler. En este proceso se produce una mezcla muy trabajable que puede extenderse mediante una maquinaria sin rodillo de compactación y que cuando se enfría es muy resistente a la deformación, aumentando así el módulo y haciendo la mezcla más rígida $[2,4,5]$.

En ese orden de ideas, es válido también preguntar sobre el efecto de la lluvia ácida en el asfalto como cementante: jes posible que se cambien las propiedades químicas del cemento asfalto bajo el efecto de la lluvia ácida mediante procesos de absorción u otro tipo de procesos más complejos?, ¿serían buenos los efectos en este caso o contribuirían al daño que el ácido sulfúrico causa sobre los agregados?

Figura 3.

Efecto del contenido de azufre sobre el ensayo Marshall. Fuente: Asphalt Machineries and Equipment

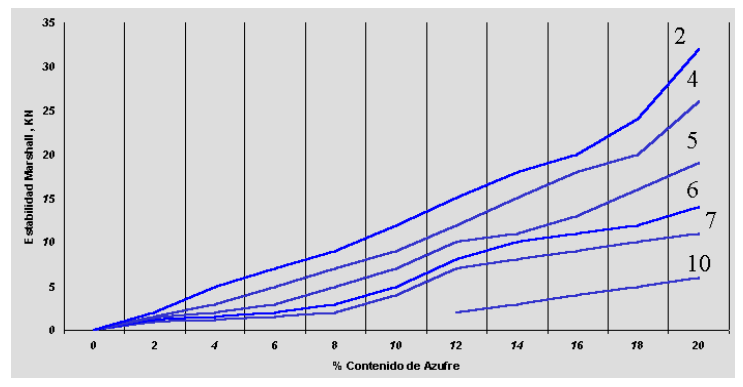




\section{Recomendaciones}

Como la lluvia ácida puede incidir en el comportamiento mecánico de un concreto asfáltico, es necesario evaluar el grado de afectación en el tiempo sobre las estructuras que usan este tipo de pavimento .

Pero, dado que su evaluación en campo tardaría mucho tiempo y la identificación de la misma en tiempo real no es la más sencilla, se recomienda evaluar dichos efectos en el laboratorio.

La herramienta más adecuada para llevar a cabo estos estudios es el "equipo de carga acelerada", con el cual se llevan a cabo pruebas aceleradas sobre los pavimentos (simulador de modelos de carga móvil) con el objeto de caracterizar mezclas asfálticas mediante diferentes tipos de mediciones.

Con este equipo (que es como una pista de ensayos a escala reducida), se pueden simular entre otros factores las cargas cíclicas de los neumáticos sobre los pavimentos vinculando factores como temperatura controlada, número de ciclos, cargas y, para este caso en particular, lluvia ácida.

Ahora bien, para simular el efecto de la lluvia ácida en el tiempo, dado que esta causa un efecto a largo plazo, se debe construir un modelo aparente para someterlo a un aumento de la gravedad en la maquina centrifuga, y en este modelo se deben recrear las cargas sobre el pavimento, los espesores, la lluvia ácida (precipitación) e inclusive los cambios de temperatura.

Al llevar a cabo dicho ensayo, se esperaría encontrar los cambios en el módulo de la mezcla (oxidación), alteraciones en la viscosidad del asfalto, alteraciones en la adherencia, y el efecto de la lluvia ácida sobre estos factores.

El ensayo debe correrse dos veces: en la primera, básicamente, se simularán cargas, temperatura y precipitación en ausencia de ácidos (sin polución o contaminación), y en la segunda la precipitación ha de ser ácida. De esta manera se medirán características asfálticas como las antes mencionadas y se 
compararán los datos para determinar el grado de afectación del fenómeno de la lluvia ácida sobre las mezclas asfálticas en el tiempo.

Esto es enriquecedor, pues con ello se puede determinar el desempeño del concreto asfáltico en circunstancias actualmente comunes, y marcar pautas adicionales para el diseño de las mezclas asfálticas [6-8]. 


\section{Conclusiones}

Es importante que los efectos de la lluvia ácida sobre el pavimento sean considerados para el diseño y la construcción de la infraestructura vial, pues esto ayudará a una mejor proyección del rendimiento y vida útil de la carretera.

En forma concreta, determinar el efecto destructivo de la lluvia acida puede dar pie a la generación de políticas gubernamentales que protejan la inversión pública e incentivar colateralmente la protección del medio ambiente.

Pero, además de conocer la implicación de agentes ambientales como la temperatura y la precipitación (humedad), es relevante investigar cómo afectan a un pavimento los agentes químicos producidos por la polución, a fin de evitar que los efectos de la lluvia ácida se conviertan en un problema de diseño de la mezcla que repercuta en el desempeño mecánico de los pavimentos.

La lluvia ácida produce un efecto a largo plazo, por lo que el reconocimiento del mismo dependerá entonces de la medida de pluviosidad ácida, la concentración de los ácidos producto de la polución, del tiempo de contacto entre los ácidos y el asfalto, y de las cargas a que sea sometido el pavimento.

Ejecutando el ensayo con el uso del simulador a escala propuesto, pero siempre comparando diferentes grados de temperatura en presencia de lluvia ácida, se podrá determinar si el efecto de la lluvia ácida varía según los cambios de temperatura, y qué tanto.

Los problemas encontrados en los pavimentos por efecto de la lluvia ácida no se pueden considerar como fatiga, dado que si el pavimento falla, como se prevé en este documento, no será solo por causa de un envejecimiento prematuro o por daños estructurales internos derivados de una acción acelerada de agentes externos como el tráfico, la humedad y posiblemente la temperatura.

El efecto de los ácidos sobre los asfaltos, con agregados cuya composición contiene carbonato de calcio, se prevé como negativa, sin embargo, se debe estimar qué tan negativa es., 
Entre algunas preguntas que se pueden solucionar con el uso de modelos a escala en un tiempo relativamente corto, podrían destacarse las siguientes: ¿El efecto del ácido sulfúrico sobre el cemento asfáltico se puede prever fácilmente?, ¿podría ser este negativo o incluso positivo, como lo indica el comportamiento de algunos modificadores a base de azufre en los asfaltos? Además, ¿qué tan negativa o positiva podría ser?, y, por último, combinada con el efecto sobre los agregados, ¿que produce?

El efecto del agua sobre los poros del asfalto y sobre la estructura granular del pavimento se puede potenciaro negativamente por la inclusión de ácidos presentes en las precipitaciones, Y, en todo caso, es claro que la lluvia ácida puede ocasionar daños estructurales asociados al lavado de los materiales, y daños mecánicos debidos al cambio en el comportamiento del asfalto por la inclusión de agentes químicos que pueden reaccionar con la composición del concreto asfáltico. 


\section{Referencia Bibliográfica}

1. Jilei Hu, Xiaochu Wang, and Rendong Guo, "Rainfall Infiltration Influence to Asphalt Concrete Pavement", China., 2011.

2. H.A. Khalid, "A new approach for the accelerated ageing of porous asphalt mixtures. Proceedings of the Institution of Civil Engineers", Transport Issue 3, pp.171-181, 2002.

3. A. E. Sánchez Eraso, J.J. López Cueca, H.A., y Rondón Quintana, L.Á. Moreno Anselmi, Influencia del agua en mezclas densas en caliente Tipo 2 (MDC-2), Bogotá, 2010. 4. www.ecologismo.com, http://www.ecologismo. com/2011/09/26/lluvia-acida-2/.

5. S. Bhattacharjee, Use of Accelerated Loading Equipment for Fatigue Characterization of Hot Mix Asphalt in the Laboratory, Worcester Polytechnic Institute, Worcester USA, 2005. 6. http://www.fundacionypf.org.ar/ publicaciones/Tierra/contents/3_recursos_tierra/cont/recur_mineros_ region.htm, Organización de las Naciones Unidas para la Educación, la Ciencia y la Cultura. 7. http://www.e-asphalt.com/aditivos/aditivos.htm, Asphalt Machineries and Equipment. 8. www.clarindeColombia.info,http://www. clarindecolombia.info/index.php?option $=$ com_content\&view $=$ article\&id $=111$ 5:isabe-usted-sobre-la-lluvia-acida\&catid=14:amanecer\&Itemid=104 


\section{Este artículo se cita}

C. Obando, "Influencia del agua en el desempeño de los pavimentos: lluvia ácida", Investigación e Innovación en Ingenierias, vol. 5, $\mathrm{n}^{\circ} .2$, pp. 190-207., 2017 